\title{
Motzkin path models of long chain polymers in slits
}

\author{
R Brak $\dagger$, G K Iliev $\ddagger$, A Rechnitzer $\S$ and S G Whittington $\ddagger$ \\ $\dagger$ Department of Mathematics and Statistics, \\ The University of Melbourne, \\ Parkville, Victoria 3010, Australia \\ $\ddagger$ Department of Chemistry, University of Toronto, \\ Toronto, Canada M5S 3H6 \\ $\S$ Department of Mathematics, University of British Columbia \\ Vancouver, Canada V6T-1Z2
}

\begin{abstract}
We consider Motzkin path models for polymers confined to a slit. The path interacts with each of the two confining lines and we define parameters $a$ and $b$ to characterize the strengths of the interactions with the two lines. We consider the cases where (i) each vertex in a confining line contributes to the energy and (ii) where each edge in a confining line contributes to the energy.

For the vertex and edge versions of Motzkin paths we can find the generating functions and give rigorous explicit expressions for the free energy at some special points in the $(a, b)$-plane, and asymptotically (ie for large slit widths) elsewhere. We find regions where the force between the lines is long-range and repulsive, short-range and repulsive and short-range and attractive. Our results indicate that the general form of the phase diagram is model independent, and similar to previous results for a Dyck path model, although the details do depend on the underlying configurational model.

We also contrast the method used here to find the generating function with the transfer matrix and heap methods.
\end{abstract}

PACS numbers: 05.50.+q, 05.70.fh, 61.41.+e 


\section{Introduction}

The problem of long polymer molecules in a good solvent confined in one or more dimensions by parallel plates has been studied by a variety of different path models (DiMarzio \& Rubin 1971, Brak et al. 2005, Janse van Rensburg et al. 2006). The behaviour of such a system is both rich and interesting. When the plates are non-attractive, the polymer's entropy is reduced due to configurational restrictions leading to a repulsive force on the plates. The repulsive nature of this force is unchanged if one of the plates is attractive to the polymer, but its magnitude is lessened by the energetic contributions of monomers in the attractive plate. This behaviour is related to the phenomenon of steric stabilization, where a colloidal dispersion is stabilized by a polymer which cannot adsorb onto the surfaces of its particles. If instead we consider a polymer confined between two attractive plates, the nature of the net force will depend on the distance between the plates. At short distances, the loss of entropy dominates, resulting in a repulsive force. At large distances, the force on the plates becomes attractive as the polymer tries to maximize the number of monomers in the plates. This results in the phenomenon of sensitized flocculation in the colloidal dispersion.

Most previous work was concerned with the self-avoiding and random walk models (DiMarzio \& Rubin 1971, Wall et al. 1977, Wall et al. 1978). The complexity of the self-avoiding walk model means that only qualitative results are available rigorously (Hammersley and Whittington 1985, Janse van Rensburg et al. 2006). For the case where the polymer has no interaction with the confining plates we write $c_{n}(w)$ for the number of self-avoiding walks on the simple cubic lattice $\mathbb{Z}^{3}$ starting at the origin and required to have the $z$-coordinate of each vertex within the slab $0 \leq z \leq w$. It is known that the limit

$$
\lim _{n \rightarrow \infty} n^{-1} \log c_{n}(w) \equiv \kappa(w)
$$

exists. In addition it has been shown that

(i) $\kappa(w)$ is strictly monotone increasing in $w$, and

(ii) $\lim _{w \rightarrow \infty} \kappa(w)=\kappa$

where $\kappa$ is the connective constant of the lattice (Hammersley \& Whittington 1985). 
Other results involving $\kappa(w)$ are very limited. In the non-interactive regime, the value of $\kappa(w)$ is known exactly only for small values of $w$ in two dimensions (Wall et al. 1977, Wall et al. 1978). When the polymer interacts with either of the planes, some numerical results are available (Janse van Rensburg et al. 2005) and there are a few rigorous results about the free energy which establish bounds on the region of the $(a, b)$-plane where the force is repulsive (Janse van Rensburg et al. 2006).

If one uses a simpler configurational model the situation improves. In a classic paper, DiMarzio and Rubin (1971) studied a random walk on a regular lattice confined between two parallel planes. They considered both the attractive and nonattractive regimes but, for the attractive case, they only considered the situation where the polymer interacts equally with both planes. They were able to determine the free energy of the polymer and tension on the planes analytically for this model.

Directed path models can be treated combinatorially. The directedness of these models allows one to determine the generating function using several different factorization schemes. In the cases of simple models, such as Dyck and Motzkin paths, most of the results which follow are analytic. Although the configurational models are simple enough to yield many analytical results, they still have enough structure to capture the basic physics of the problem. This makes them both useful and valuable tools.

The simplest of this class of models is Dyck paths. This model is closely related to the ballot path problem and to the Catalan numbers. Dyck paths are defined as all paths which start at $(0,0)$ and are made up of steps in the $(1,1)$ and $(1,-1)$ directions with no steps below the line $y=0$ and with their final vertex in the line $y=0$. For Dyck paths in a slit of width $w$ there is an additional condition that no vertex should lie above the line $y=w$.

A recent paper by Brak et al. (2005) focused on several models related to Dyck paths

(i) loops - paths which start and end in the bottom surface i.e. Dyck paths,

(ii) bridges - paths which start in the bottom surface and end in the top surface,

(iii) tails - paths which start in the bottom surface and end anywhere within the slit.

The treatment of each of the three cases is very similar. Although each of them 


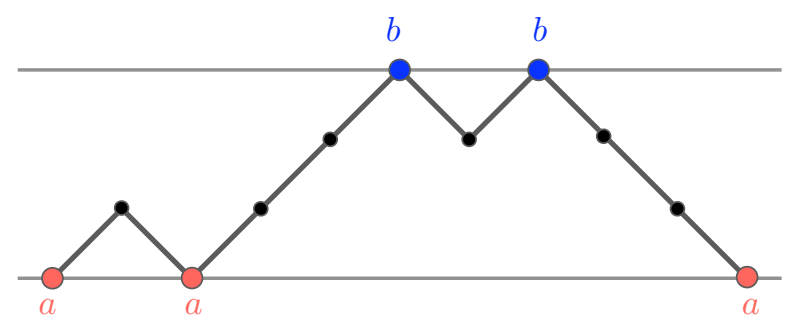

Figure 1. An example of a Dyck path with lower and upper vertex surface weights, $a$ and $b$ respectively.

results in a different generating function, their singularity structure is identical and so is their thermodynamic limit. This result is not unexpected, as there are the same number of paths in each of the three cases, to exponential order. A general result (Hammersley et al. 1982) implies that the end point of the path cannot determine its thermodynamics. For this reason, we will only consider the case of loops for the rest of our models since it is combinatorially simpler.

The notation used for all of the models considered here will be similar to that introduced by Brak et al. (2005). The confining planes will be represented by the lines $y=0$ and $y=w$ for the lower and upper planes, respectively. We shall consider $z$ to be conjugate to the number of edges, $n$, in the path. Vertices in the line $y=0$ will have an associated weight $a$, whereas vertices in the line $y=w$ will be weighted by $b$.

When they considered the case of Dyck paths, Brak et al. were able to determine a functional recurrence for the generating functions at various slit widths. The recurrence considers Dyck paths in a slit of width $w$, and replaces any vertices in the line $y=w$ by a zig-zag pattern of width 1 . This produces a Dyck path that fits in a slit of width $w+1$. We can write this as:

$$
\begin{aligned}
D_{1}(a, b, z) & =\frac{1}{1-a b z^{2}} \\
D_{w+1}(a, b, z) & =D_{w}\left(a, \frac{1}{1-b z^{2}}, z\right), \quad w \geq 1 .
\end{aligned}
$$

By iterating $D_{w}(a, b, z)$ they were able to build up the generating functions for small values of $w$ and note that they were rational functions. It was found that the numerators and denominators of $D_{w}(a, b, z)$ satisfy a recurrence relation whose solution determines the functional dependence of the generating function on 
$w$. From this generating function, they were able to extract the asymptotic form of the free energy for large $w$ and determine the phase diagram for the model. Furthermore, they obtained expressions for the form of the tension on the surfaces for large $w$ throughout the interaction space, noting regions of long- and shortranged repulsion along with a region of short-ranged attraction. These attractive and repulsive regions were separated by a curve of zero force which had a simple analytical form. The authors also consider the case where $w \rightarrow \infty$ and its relation to the half-plane problem. The process involves two limits which do not commute. In order to recover the half-plane result, one must let $w \rightarrow \infty$ before $n \rightarrow \infty$ so that the path never "sees" the upper surface. If one interchanges the order of these two limits, then even when $w \rightarrow \infty$ one finds that the results still depend on both interaction parameters even though the model has been reduced to a single surface problem. The directed path models considered in this paper are closely related to
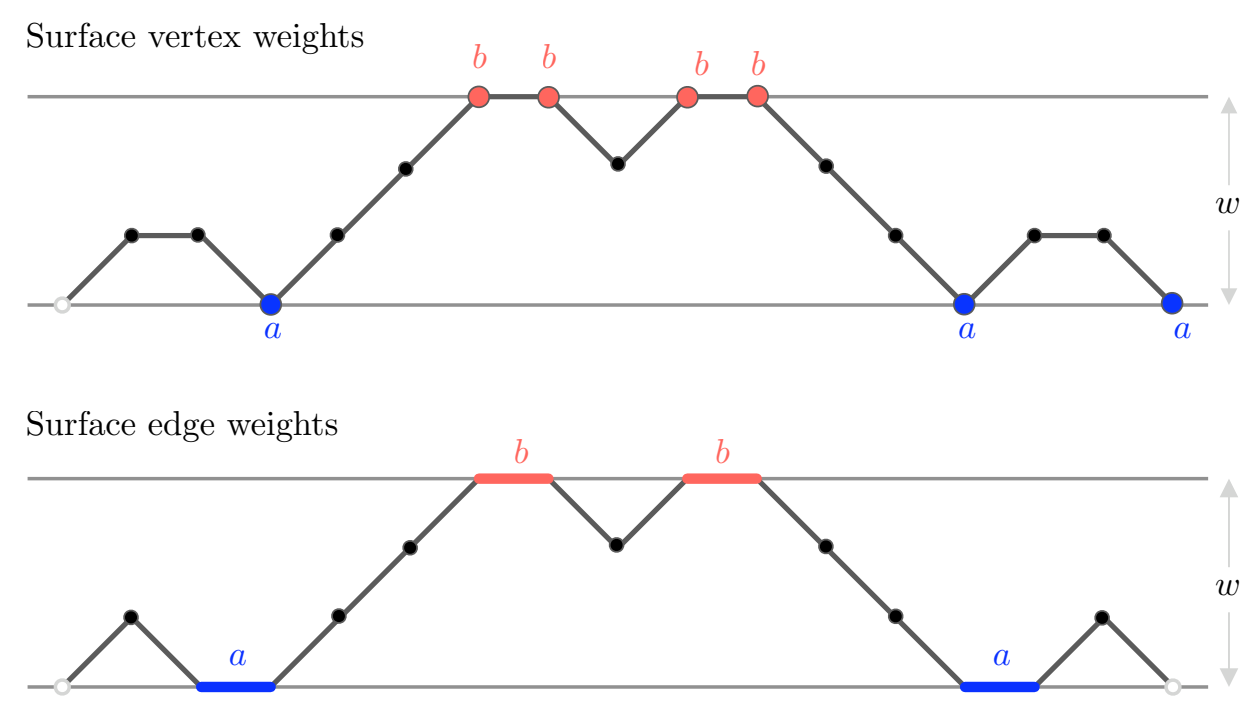

Figure 2. Motzkin paths with lower and upper vertex and edge surface weights, $a$ and $b$ respectively.

the models of Motzkin paths. Motzkin paths are paths on $\mathbb{Z}^{2}$ that start and end in the line $y=0$, have no vertices below this line and have steps in the $(1,0),(1,1)$ and $(1,-1)$ directions. Since we are only considering all such paths up to a translation, we will only look at paths which start at $(0,0)$. As with Dyck paths, the models considered here have a further restriction that all vertices must lie within a slit of 
width $w$.

\section{Motzkin Paths}

Motzkin paths are a class of paths closely related to Dyck paths. In addition to the steps $(1,1)$ and $(1,-1)$, Motzkin paths also include the step $(1,0)$. The inclusion of a horizontal step allows the path to have every vertex in the surface, and also to have edges in the surfaces. In the vertex version of the model, the interactions $a$ and $b$ with the lower and upper surfaces are associated with vertices in the lines $y=0$ and $y=w$, respectively.

\subsection{Vertex Model}

For the vertex model we can write the half-plane path generating function as,

$$
M_{w}^{v}(a, b, z)=\sum_{\omega \in \Omega_{w}} a^{u_{1}(\omega)} b^{u_{2}(\omega)} z^{n(\omega)}
$$

where $\Omega_{w}$ represents the set of all Motzkin paths in a slit of width $w$, and $u_{1}(\omega)+1$ (ie the first vertex is not weighted) and $u_{2}(\omega)$ are the numbers of vertices in the lines $y=0$ and $y=w$ for a path $\omega$, respectively. $n(\omega)$ is the number of edges in $\omega$.

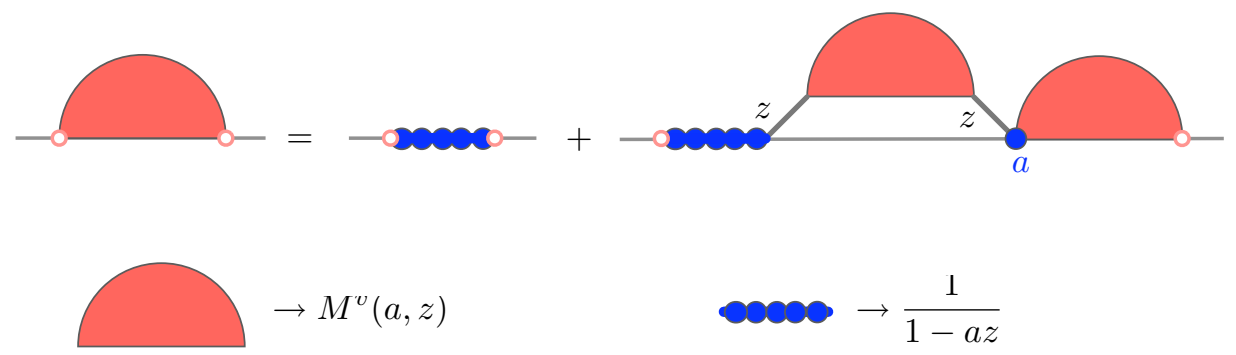

The case of a path interacting with a single surface can be considered as a special case of the slit problem. If we let $w \rightarrow \infty$ before letting $n \rightarrow \infty$ we get the half-plane problem, which implies that $u_{2}(\omega)=0$. The factorization of Motzkin paths (Motzkin 1948, Schröder 1870) in this situation is well known and lends itself to several interesting factorization methods. Since we are studying loops, the waspwaist factorization is particularly useful. If we keep track of the number of steps that the path takes before it steps out of the bottom surface, we get the following theorem. 
Theorem 1. The half-plane Motzkin path generating function (2.1) factorizes as

$$
M^{v}(a, z)=\frac{1}{1-a z}\left(1+a z^{2} M^{v}(1, z) M^{v}(a, z)\right) .
$$

and hence

$$
M^{v}(a, z)=\frac{2}{2-a z-a+a \sqrt{1-2 z-3 z^{2}}} .
$$

Proof. This result is a well known generalisation of the standard Motzkin and Dyck path factorization (Janse van Rensburg 2000). The set of half-plane Motzkin paths partitions into two disjoint subsets: 1) The paths have no up step - generated by $(1-a z)^{-1}$, or 2 ) the paths have at least one up step. In the latter case, following the first down step to $y=0$ can be any Motzkin path. Prior to the first up step can be any number of horizontal step, thus case 2) corresponds to $a z^{2} M^{v}(1, z) M^{v}(a, z) /(1-a z)$

There is a square-root singularity at $z_{1}=1 / 3$ and a simple pole from the zero of the denominator, $z_{2}(a)$. For values of $a<3 / 2$, the square-root singularity is dominant while for $a>3 / 2$, the simple pole is dominant. At $a=3 / 2$, the two coalesce, identifying the critical point for the model. The dominant singularity determines the free-energy, $\kappa(a)$, of the system via:

$$
\kappa(a)=-\log z_{c}(a)
$$

where $z_{c}(a)$ represents the dominant singularity of the model.

For paths in a strip of width $w$, we can obtain the exact expressions for the partition functions for small values of $w$. The factorization is very similar to that used in the half-plane problem, but we impose a height restriction on the paths. The surface vertex weighted Motzkin path generating function is given by the following theorem.

Theorem 2. The following statements are equivalent.

(i) $M_{w}^{v}(a, b, z)$ is the generating function (2.1) for surface vertex weighted Motzkin paths.

(ii) $M_{w}^{v}(a, b, z)$ factorizes into the form,

$$
M_{w}^{v}(a, b, z)=\frac{1}{1-a z}\left(1+a z^{2} M_{w-1}^{v}(1, b, z) M_{w}^{v}(a, b, z)\right) .
$$


with initial (width one) generating function,

$$
M_{1}^{v}(a, b, z)=\frac{1-b z}{1-a z-b z} .
$$

(iii) $M_{w}^{v}(a, b, z)$ is a rational function

$$
M_{w}^{v}(a, b, z)=\frac{P_{w}^{v}(a, b, z)}{Q_{w}^{v}(a, b, z)}
$$

where the polynomials $P_{w}^{v}(a, b, z)$ and $Q_{w}^{v}(a, b, z)$ satisfy the same three term recurrence relation,

$$
T_{w+2}=(1-z) T_{w+1}-z^{2} T_{w}, \quad w \geq 0
$$

(ie replace $T_{k}$ with $P_{k}^{v}$ or $Q_{k}^{v}$ ) but with different initial conditions

$$
P_{0}^{v}=b, \quad P_{1}^{v}=1-b z, \quad \text { and } \quad Q_{0}^{v}=a+b-a b(1+z), \quad Q_{1}^{v}=1-z(a+b)(2
$$

(iv) $M_{w}^{v}(a, b, z)$ satisfies the equation

$$
M_{w}^{v}(a, b, z)=\frac{1}{1-a z-a z^{2} M_{w-1}^{v}(1, b, z)} \quad w \geq 2 .
$$

Iterating this gives:

$$
M_{w}^{v}(a, b, z)=\frac{1}{1-a z-a z^{2} F_{w-1}^{v}\left(M_{1}^{v}(1, b, z)\right)}, \quad w \geq 2
$$

where $F_{w}^{v}(a)$ satisfies the functional equation

$$
F_{w+1}^{v}(a)=F_{w}^{v}\left(\frac{1}{1-z-a z^{2}}\right), \quad w \geq 2
$$

with initial condition

$$
F_{2}^{v}(a)=\frac{1}{1-z-a z^{2}}
$$

and $F_{1}^{v}(a)=\frac{1-b z}{1-z-b z}$.

Equation (2.12) is the Motzkin path analogue of the Dyck path functional equation (1.3). For the proofs of the equivalences we only show one of the directions, the other following by simple reversal of the argument.

Proof. ii) if $i$ ): This result is a simple generalization of Theorem 1. The set of all surface vertex weighted Motzkin paths paths partitions into the following two disjoint subsets (note, the zero length path is in the set):

(i) The path has no up step. This set is generated by $(1-a z)^{-1}$. 
(ii) The path has at least one up step. In this case the path following the first down step to $y=0$ can be any Motzkin path of width $w$. Between the first up step from $y=0$ and the first down step to $y=0$ can be any Motzkin path of width $w-1$ but no $a$ weights. This subset is generated by $a z^{2} M_{w-1}^{v}(1, b, z) M_{w}^{v}(a, b, z) /(1-a z)$.

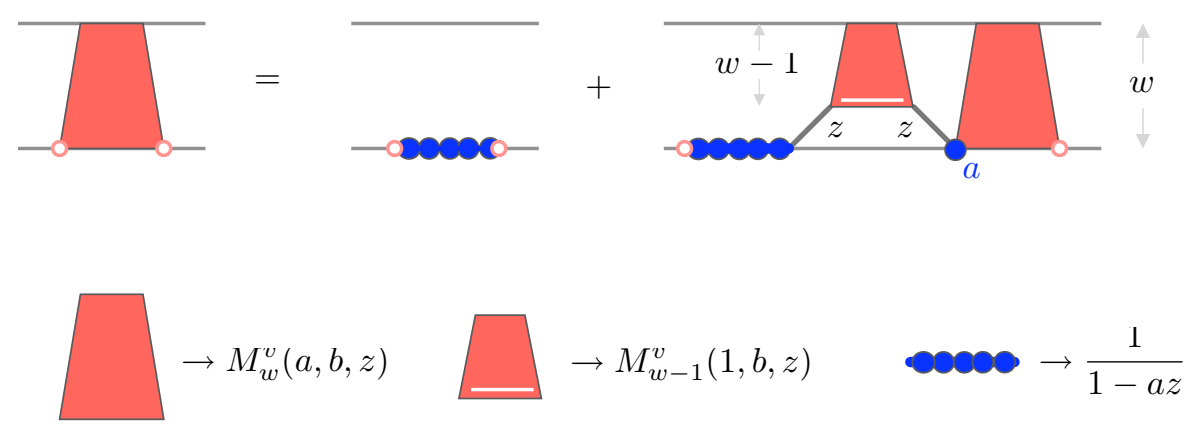

iii) if ii): Solve (2.5) for $M_{w}^{v}(a, b, z)$,

$$
M_{w}^{v}(a, b, z)=\frac{1}{1-a z-a z^{2} M_{w-1}^{v}(1, b, z)}
$$

and then substitute (2.7) to give

$$
\frac{P_{w+1}}{Q_{w+1}}=\frac{\bar{Q}_{w}}{(1-a z) \bar{Q}_{w}-a z^{2} \bar{P}_{w}}
$$

where $\bar{Q}_{w}=Q_{w}(1, b, z)$ and $\bar{P}_{w}=P_{w}(1, b, z)$. If we divide out any common factors on the left hand side of the above equation, the numerator and denominator of the right hand side could still have common factors. If we take these into account these cancel in the final analysis so we are able to write

$$
\begin{aligned}
P_{w+1} & =\bar{Q}_{w} \quad \text { and } \\
Q_{w+1} & =(1-a z) \bar{Q}_{w}-a z^{2} \bar{P}_{w} .
\end{aligned}
$$

Putting $a=1$, we obtain the recurrence relation

$$
\bar{Q}_{w+1}=(1-z) \bar{Q}_{w}-z^{2} \bar{Q}_{w-1}
$$

and the same recurrence relation for $\bar{P}_{w}$ because of (2.16), but with different initial conditions. To get the recurrence relation for $Q_{w}$ take the same linear combination 
as (2.18), but use (2.17) (and (2.16)) to give

$$
\begin{aligned}
-Q_{w+2}+(1-z) Q_{w+1}-z^{2} Q_{w}= & (1-a z)\left(-\bar{Q}_{w+1}+(1-z) \bar{Q}_{w}-z^{2} \bar{Q}_{w-1}\right) \\
& -a z^{2}\left(-\bar{Q}_{w}+(1-z) \bar{Q}_{w-1}-z^{2} \bar{Q}_{w-2}\right)
\end{aligned}
$$

and hence $Q_{w}$ satisfies

$$
Q_{w+1}=(1-z) Q_{w}-z^{2} Q_{w-1}
$$

and similarly for $P_{w}$. The initial conditions (2.9) are then obtained by comparing (2.15) with $M_{1}^{v}$ and $M_{2}^{v}$.

iv) if iii): To get iv) from iii) we need $M_{w+1}^{v}(1, b, z)$ from (2.14). Thus, put $a=1$ in $(2.14)$ to give

$$
M_{w+1}^{v}(1, b, z)=\frac{1}{1-z-z^{2} M_{w}^{v}(1, b, z)}
$$

which is the standard Jacobi continued fraction form (Wall 1948) with the functional form (2.12) and initial condition (2.13).

The closest singularity to the origin on the real axis determines the behaviour of the thermodynamic system. Since the partition functions are rational functions, all singularities will arise from zeros of the denominators. While we can calculate these zeros explicitly for small values of $w$, in order to obtain the functional dependence of the zeros on the width of the slit, we need to solve the recurrence relation. The general form of $Q_{w}^{v}(a, b, z)$ can be written as:

$$
Q_{w}^{v}(a, b, z)=A(a, b, z) \lambda_{1}(z)^{w}+B(a, b, z) \lambda_{2}(z)^{w}
$$

where $\lambda_{2}(z) \geq \lambda_{1}(z)$ for all $z \in[0,1 / 3]$. We can carry out the same procedure for the polynomials in the numerator and obtain a similar expression for $P_{w}^{v}(a, b, z)$ which involves the same $\lambda_{1}(z)$ and $\lambda_{2}(z)$ with different coefficients. It is also important to note that the only $w$-dependence in the above expression occurs in the exponents of the two $\lambda_{i}(z)$ 's. Hence we can write

$$
\frac{P_{w}}{Q_{w}}=\frac{C \lambda_{1}^{w}+D \lambda_{2}^{w}}{A \lambda_{1}^{w}+B \lambda_{2}^{w}},
$$

where $A, B, C$ and $D$ are polynomials in $a, b$ and $z$.

The change of variables:

$$
q^{2}=\frac{\lambda_{1}(z)}{\lambda_{2}(z)}
$$


can be used to simplify the partition functions and their denominators. The inverse of this substitution has the rather simple form:

$$
z=\frac{q}{1+q+q^{2}}
$$

With this substitution we can write

$$
\frac{P_{w}}{Q_{w}}=\frac{C q^{2 w}+D}{A q^{2 w}+B},
$$

and so we get the following theorem.

Theorem 3. The zeros of the denominators of $M_{w}^{v}(a, b, z)$, that is zeros of $Q_{w}^{v}(a, b, z)$, in the $q$ variable, (2.25) are solutions of the degree $2 w+4$ equation

$$
q^{2 w}=\frac{\left(a q^{2}-q^{2}+a q-q-1\right)\left(b q^{2}-q^{2}+b q-q-1\right)}{\left(q^{2}-a q+q-a+1\right)\left(q^{2}-b q+q-b+1\right)} .
$$

Similarly the zeros of the numerators of $M_{w}^{v}$ are given by

$$
q^{2 w+2}=\frac{\left(b q^{2}-q^{2}+b q-q-1\right)}{\left(q^{2}-b q+q-b+1\right)} .
$$

Note that both of these equations are symmetric in $q \leftrightarrow 1 / q$.

2.1.1. Location of singularities Before we can describe the phase diagram of the model we need to prove some facts about the locations of the singularities of the generating function and so the zeros of equation (2.27).

Lemma 2.1. For all $a, b \geq 0$ and $w \geq 1$, the dominant singularity $z_{c}$ lies on the positive real axis. Then either $q_{c}$ lies on the unit circle or on the positive real line. Further, if $q_{c}=e^{i \theta}$, then $z_{c}=(1+2 \cos \theta)^{-1} \in[1 / 3, \infty)$, while if $0 \leq q_{c}<\infty$, then $z_{c} \in[0,1 / 3]$.

Proof. The generating function, $M_{w}^{v}(a, b, z)$ is a positive term power series and so its dominant singularity lies on the positive real axis (Pringsheim's theorem). Since $z_{c}=q_{c} /\left(1+q_{c}+q_{c}^{2}\right)$, it follows that $q_{c}+1+1 / q_{c} \in \mathbb{R}$. Substituting $q=r e^{i \theta}$ we obtain

$$
r e^{i \theta}+\frac{1}{r} e^{-i \theta}+1=\left(r-\frac{1}{r}\right) e^{i \theta}+\frac{2}{r} \cos \theta+1 \in \mathbb{R}
$$

Hence either $r=1$ or $\theta$ is an integer multiple of $\pi$ and so $q_{c}$ either lies on the unit circle or on the non-negative real line (since $z_{c} \geq 0$ ). The result follows. 
Lemma 2.2. The radius of convergence, $z_{c}(a, b, w)$, of $M_{w}^{v}$ is a monotonic nonincreasing function of $a$ and $b$. Hence for a fixed $w$ and any $a, b \leq 3 / 2$ it is bounded above by its value at $a, b=3 / 2$.

Proof. The generating function $M_{w}^{v}$ is a power series in $z$ with coefficients that are positive polynomials in $a$ and $b$. It follows that its radius of convergence is a monotonic non-increasing function of $a$ and $b$.

Lemma 2.3. At the point $(a, b)=(3 / 2,3 / 2), q=1$ (and so $z=1 / 3$ ) is a double pole of $M_{w}^{v}$. Further, it is the dominant singularity irrespective of $w$.

Proof. At $a=b=3 / 2$, equations (2.27) and (2.28) for the denominator and numerator zeros may be written as (respectively):

$$
\begin{aligned}
& (2 q+1)^{2} q^{2 w}-(q+2)^{2}=0 \\
& (2 q+1) q^{2 w+2}-(q+2)=0
\end{aligned}
$$

We see that $q=1$ is a zero of both equations. Differentiating these expressions repeatedly with respect to $q$ and setting $q=1$ shows that $q=1$ is a triple zero of the denominator and a simple zero of the numerator. Hence $q=1(z=1 / 3)$ is a double pole of $M_{w}^{v}$.

If the singularity at $q=1$ is not the dominant singularity, it follows from Lemma 2.1 that there must be a singularity at $0 \leq q_{c}<1$. If we substitute this value into equation(2.27) at $a, b=3 / 2$ we find

$$
q_{c}^{2 w}=\left(\frac{q_{c}+2}{2 q_{c}+1}\right)^{2} \in[0,1)
$$

However in the range $0 \leq x<1$, the function $\frac{x+2}{2 x+1}>1$. Hence there is no $q_{c} \in[0,1)$ and so $q_{c}=1$.

Combining the previous two lemmas we obtain

Lemma 2.4. For $a, b \leq 3 / 2$ the dominant singularity $q_{c}$ of $M_{w}^{v}$ lies on the unit circle.

We make use of the above lemma in the next section to determine large $w$ asymptotic expressions for $q_{c}$ and $z_{c}$. Additionally there is a curve in the plane along which the dominant singularity is independent of $w$. This is a line of zero force in the phase diagram. 
Lemma 2.5. Along the curve $(a-1)(b-1)(a+b+1)=1$ (with $a \geq b$ ), the dominant singularity is independent of $w$ and is given by

$$
q_{c}=\frac{1-a+\sqrt{a^{2}+2 a-3}}{2(a-1)} \quad \text { and } \quad z_{c}=\frac{1-a+\sqrt{a^{2}+2 a-3}}{2 a} .
$$

If $b>a$ then similar expressions hold but with $b$ in place of $a$.

Proof. If $q_{c}$ is a zero of equation (2.27) independent of $w$ then both the denominator and numerator of the right-hand side must be simultaneously zero; this gives two equations in $a, b$ and $q$. Eliminating $q$ from these equations gives the following equation for $a$ and $b$ :

$$
a b(3-2 a)(3-2 b)((a-1)(b-1)(a+b+1)-1)=0 .
$$

One can exclude the cases $a$ or $b=0$ and $a$ or $b=3 / 2$ in much the same manner as the proof of Lemma 2.3. Along the curve $(a-1)(b-1)(a+b+1)=1$ with $a>b$ there are two $w$-independent real positive solutions for $q$ :

$$
q=\frac{1-a+\sqrt{a^{2}+2 a-3}}{2(a-1)}, \frac{a-1+\sqrt{a^{2}+2 a-3}}{2} .
$$

For $a>3 / 2$ (which corresponds to the section of curve with $a>b$ ), the first of these solutions is smaller. We now need to show that there is no smaller positive solution of equation (2.27).

Rearrange equation (2.27) to give

$$
\begin{aligned}
& q^{2 w}\left(q^{2}-a q+q-a+1\right)\left(q^{2}-b q+q-b+1\right)= \\
& \left(a q^{2}-q^{2}+a q-q-1\right)\left(b q^{2}-q^{2}+b q-q-1\right)
\end{aligned}
$$

For $q$ small and positive and $a>b$ on the curve, the right-hand side is positive, while the left-hand side is negative; there cannot be a real solution until one side changes sign. The right-hand has its smallest positive zero (in $q$ ) at $q=\frac{1-a+\sqrt{a^{2}+2 a-3}}{2(a-1)}$. This is precisely the point at which the left-hand side also has its smallest positive zero (in $q$ ). Hence this value of $q$ is the smallest positive solution of (2.27) along the curve with $a>b$. The case $a<b$ follows by symmetry. When $a=b$ along the curve they are equal to $3 / 2$ and so correspond to Lemma 2.3. 
2.1.2. Regions in the $(a, b)$ plane The behaviour of the problem defines several regions in the $(a, b)$ plane. Within and along the boundaries of these regions, there are several points where the expression for the dominant singularity of $M_{w}^{v}(a, b, z)$ in (2.27) simplifies. For the vertex model of Motzkin paths, these points include $(1,1),(3 / 2,1),(1,3 / 2)$ as well as the points along the curve:

$$
(a-1)(b-1)(a+b+1)=1
$$

which includes the point $(3 / 2,3 / 2)$. The value $a_{c}=3 / 2$ represents the critical point in the half-plane problem. Furthermore, because of the $(a, b)$ symmetry in $(2.27)$, we will only consider the cases where $a \geq b$. Behaviour in the other regime can be obtained by identical arguments and the results are the same up to the interchange of $a$ and $b$.

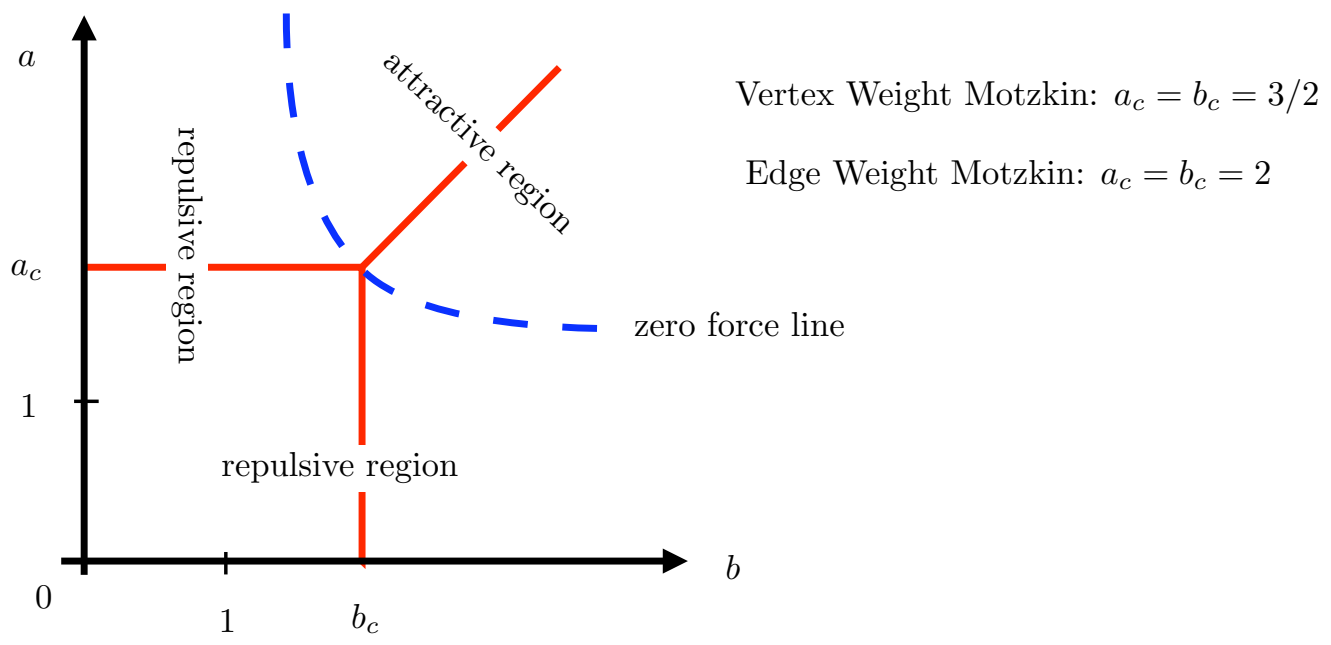

Figure 3. Phase and force diagram for the Motzkin edge and vertex model.

We first consider the interior of the square, $S$, defined by the vertices $\left(s_{1}, s_{2}\right)$, where $s_{1}, s_{2} \in\{0,3 / 2\}$. The point $(1,1)$ represents a special point in this region. At $(1,1)$ the expression for the zeros of the denominator in $(2.27)$ simplifies to:

$$
q^{2 w}=\frac{1}{q^{4}}
$$

which clearly represent $(2 w+4)$-th roots of unity. The general solution can be written as:

$$
q_{c}(1,1, w)=\exp \left(i \frac{2 n \pi}{2 w+4}\right), \quad n=0 \ldots 2 w+3 .
$$


Under the transformation (2.25), this becomes:

$$
z_{c}(1,1, w)=\frac{1}{1+2 \cos \left(\frac{\pi}{w+2}\right)}
$$

where we have used an argument similar to that of Hammersley and Whittington (1985) to show that $\kappa(w)$ is a strictly monotone increasing function in $w$. For our system this implies that $\kappa(w)<\log (3)$ for all finite $w$. According to (2.4) this is equivalent to $z_{c}(w)>1 / 3$, which implies that $n \neq 0$. Since the closest singularity to the origin dominates, we conclude that $n=1$.

We note that we can explicitly exclude $q=1($ ie $z=1 / 3)$ as a pole of $M_{w}^{v}$ by showing that $q=1$ is a simple zero of both the numerator and denominator of equation $(2.26)$ (except at the point $(a, b)=(3 / 2,3 / 2))$. Hence $z_{c}$ is determined by the next zero of equation (2.27). At $(a, b)=(3 / 2,3 / 2) q=1$ is a multiple zero of the denominator but a simple zero of the numerator and so the dominant singularity is $q=1$ ie $z_{c}=1 / 3$.

At $(a, b)=(1,1)$, the asymptotic behaviour of the singularity and the free energy in inverse powers of $w$ are:

$$
\begin{aligned}
& z_{c}(1,1, w)=\frac{1}{3}+\frac{\pi^{2}}{9 w^{2}}-\frac{4 \pi^{2}}{9 w^{3}}+O\left(\frac{1}{w^{4}}\right) \\
& \kappa(1,1, w)=\log (3)-\frac{\pi^{2}}{3 w^{2}}+\frac{4 \pi^{2}}{3 w^{3}}+O\left(\frac{1}{w^{4}}\right) .
\end{aligned}
$$

Since the model is a random walk in $(1+1)$ dimensions the length exponent perpendicular to the preferred direction is $1 / 2$ and the first correction term in $\kappa(1,1, w)$ is proportional to $w^{-2}$.

We can also look at points away from $(1,1)$ that are still in the interior of the square $S$. For solutions to (2.27) we propose the Ansatz that the critical value of $q$ takes the form:

$$
\begin{aligned}
q_{c}(a, b, w) & =\exp \left(i \frac{2 \pi}{2 w+\delta(a, b)}\right) \\
& \times\left[1+\frac{C_{1}}{w}+\frac{C_{2}}{w^{2}}+\frac{C_{3}}{w^{3}}+\frac{C_{4}}{w^{4}}+O\left(\frac{1}{w^{5}}\right)\right] .
\end{aligned}
$$

By using this form in (2.27) and expanding in $1 / w$, we obtain:

$$
\delta(a, b)=\frac{2(3 a-4 a b+3 b)}{(2 a-3)(2 b-3)},
$$


and

$$
C_{1}=C_{2}=C_{3}=0 \quad \text { while } \quad C_{4}=C_{4}(a, b) \neq 0 .
$$

Hence

$$
\begin{aligned}
z_{c}(a, b, w) & =\left[\frac{1}{1+2 \cos \left(\frac{2 \pi}{2 w+\delta(a, b)}\right)}\right]\left[1+\frac{C_{4}(a, b)}{w^{4}}+O\left(\frac{1}{w^{5}}\right)\right] \\
& =\frac{1}{3}+\frac{\pi^{2}}{9 w^{2}}-\frac{2 \pi^{2}(3 a-4 a b+3 b)}{9(2 a-3)(2 b-3) w^{3}}+O\left(\frac{1}{w^{4}}\right)
\end{aligned}
$$

and

$$
\begin{aligned}
\kappa(a, b, w) & =-\log \left[z_{c}(a, b, w)\right] \\
& =\log (3)-\frac{\pi^{2}}{3 w^{2}}+\frac{2 \pi^{2}(3 a-4 a b+3 b)}{3(2 a-3)(2 b-3) w^{3}}+O\left(\frac{1}{w^{4}}\right) .
\end{aligned}
$$

The term in $1 / w^{2}$ is independent of $(a, b)$ and the first $(a, b)$ dependence occurs in the $w^{-3}$ term. Also, the form of $\delta(a, b)$ and the asymptotics of $z_{c}$ and $\kappa$ suggest that the proposed solution in only valid for the interior of the square $S$, as it breaks down for values of $a=3 / 2$ or $b=3 / 2$.

The situation along the boundary of $S$ is more complicated. If we consider $L=\{(a, b) \mid a=3 / 2,0<b<3 / 2\}$, then even at the point $(3 / 2,1)$ the condition in (2.27) remains sufficiently complicated that we cannot determine the exact solution. Instead, we write $z_{c}(3 / 2,1, w)$ as a power series in $1 / w$. Clearly the constant term is $1 / 3$ and we can compute the coefficients of the first few powers of $1 / w$ to obtain:

$$
\begin{aligned}
& z_{c}(3 / 2,1, w)=\frac{1}{3}+\frac{\pi^{2}}{36 w^{2}}-\frac{7 \pi^{2}}{108 w^{3}}+\frac{\pi^{2}\left(3 \pi^{2}+196\right)}{1728 w^{4}}+O\left(\frac{1}{w^{5}}\right) \\
& \kappa(3 / 2,1, w)=\log (3)-\frac{\pi^{2}}{12 w^{2}}+\frac{7 \pi^{2}}{36 w^{3}}-\frac{\pi^{2}\left(\pi^{2}+196\right)}{576 w^{4}}+O\left(\frac{1}{w^{5}}\right) .
\end{aligned}
$$

Similarly, we can consider points along $L$, but away from $(3 / 2,1)$. Again, we can only build up this solution as a series, which gives:

$$
\begin{aligned}
z_{c}(3 / 2, b, w)= & \frac{1}{3}+\frac{\pi^{2}}{36 w^{2}}+\frac{(4 b+3) \pi^{2}}{108(2 b-3) w^{3}} \\
& +\frac{\pi^{2}\left[3 \pi^{2}(2 b-3)^{2}+4(4 b+3)^{2}\right]}{1728(2 b-3)^{2} w^{4}}+O\left(\frac{1}{w^{5}}\right) \\
\kappa(3 / 2, b, w)= & \log (3)-\frac{\pi^{2}}{12 w^{2}}-\frac{(4 b+3) \pi^{2}}{36(2 b-3) w^{3}} \\
& -\frac{\pi^{2}\left[\pi^{2}(2 b-3)^{2}+4(4 b+3)^{2}\right]}{576(2 b-3)^{2} w^{4}}+O\left(\frac{1}{w^{5}}\right)
\end{aligned}
$$


These forms no longer hold at the point $(3 / 2,3 / 2)$; as noted above $z_{c}(3 / 2,3 / 2, w)=1 / 3$ independent of $w$.

Outside the square $S$ the behaviour of the system changes completely. Without essential loss of generality, consider $a \geq b$. When $w \rightarrow \infty$ we expect the free energy to be the same as that of the half-plane problem. In particular that $z_{c}<1 / 3$ for $a>a_{c}=3 / 2$. To see this we examine equation (2.27) in the $w \rightarrow \infty$ limit.

There are 3 possibilities: $|q|<1,|q|>1$ and $|q|=1$. Since equation (2.27) is invariant under $q \leftrightarrow 1 / q$, we can restrict our attention to $|q| \leq 1$. When $|q|=1$, we may write $q=e^{i \theta}$ and so $z=1 /(1+2 \cos \theta) \geq 1 / 3$. If $|q|<1$ then when $w \rightarrow \infty$, equation (2.27) reduces to

$$
\left(a q^{2}-q^{2}+a q-q-1\right)\left(b q^{2}-q^{2}+b q-q-1\right)=0 .
$$

Solving this gives the critical value of $q$ :

$$
q_{o}=\frac{1-a+\sqrt{a^{2}+2 a-3}}{2(a-1)} .
$$

Transforming this result into the $z$ variable, we get:

$$
z_{o}=\frac{1-a+\sqrt{a^{2}+2 a-3}}{2 a} .
$$

We note that if $b>a$ then we obtain the same result but with $b$ replacing $a$.

This is the expected result as it represents the value of $z_{c}$ for the adsorption of a Motzkin path at a single, impenetrable surface. There will also be an identical solution in the region where $b>a$ with all $a$ 's replaced by $b$ 's. The existence of two critical values even in the case of $w \rightarrow \infty$ is a result of the fact that we let $n \rightarrow \infty$ before letting $w \rightarrow \infty$.

For finite values of $w$, note that the left-hand side of (2.27) will include a term of the form $q_{o}^{2 w}$. As such, we propose the Ansatz:

$$
q_{c}(a, b)=q_{o}+f_{q}(a, b) q_{o}^{2 w}+O\left(q_{o}^{3 w}\right)
$$

where we can determine that:

$$
f_{q}(a, b)=\frac{a\left(a-3+\sqrt{a^{2}+2 a-3}\right)\left(2 a+b-a b-b \sqrt{a^{2}+2 a-3}\right)}{4(a-1)(a-b) \sqrt{a^{2}+2 a-3}} .
$$

In the $z$-variable, this result becomes:

$$
z_{c}(a, b, w)=z_{o}+f_{z}(a, b) q_{o}^{2 w}+O\left(q_{o}^{3 w}\right)
$$


where

$$
f_{z}(a, b)=\left(\frac{a-1}{a}\right) f_{q}(a, b) .
$$

Of particular interest will be the behaviour of $f_{z}(a, b)$, especially when we consider the forces on the surfaces. For now, it is sufficient to note that the zeros of $f_{z}(a, b)$ are given by all the points $(a, b)$ which satisfy (2.36). For these points, the form of $z_{c}(a, b, w)$ simplifies greatly and is given by the critical value of $z$ for the adsorption problem, $z_{0}$. This is consistent with the result of Lemma 2.5.

We note that the form of this solution changes when $a=b$, as $f_{q}(a, b)$, and hence $f_{z}(a, b)$ are singular along this line. For this case, equation (2.27) becomes:

$$
q^{w}= \pm \frac{\left(a q^{2}-q^{2}+a q-q-1\right)}{\left(q^{2}-a q+q-a+1\right)} .
$$

and when $w \rightarrow \infty$ the critical value of $q$ is the same as (2.52). For finite values of $w$, the proposed form of $q_{c}$ changes to:

$$
q_{c}(a, a)=q_{o}+g_{q}(a) q_{o}^{w}+O\left(q_{o}^{2 w}\right)
$$

with:

$$
g_{q}(a)=-\frac{a\left(a-3+\sqrt{\left.a^{2}+2 a-3\right)}\right.}{2(a-1) \sqrt{a^{2}+2 a-3}} .
$$

Transforming this result to the $z$ variable, we get:

$$
z_{c}(a, a, w)=z_{o}+g_{z}(a) q_{o}^{w}+O\left(q_{o}^{2 w}\right)
$$

where:

$$
g_{z}(a)=\left(\frac{a-1}{a}\right) g_{q}(a)
$$

2.1.3. Forces Given the forms of $\kappa$ in all of these regimes, we can transform these expressions into results regarding the forces exerted on the confining surfaces. The relation between the free energy and this force can be written as:

$$
\mathcal{F}(w)=\frac{\partial \kappa(w)}{\partial w}=-\frac{\partial \log \left(z_{c}(w)\right)}{\partial w}=-\frac{1}{z_{c}(w)} \frac{\partial z_{c}(w)}{\partial w} .
$$

Applying this to our results, we start with our analytical result at $(1,1)$. Here, $1 / z_{c}=2 \cos [\pi /(w+2)]+1$, which gives a force of:

$$
\mathcal{F}(w)=\frac{2 \pi}{(w+2)^{2}} \frac{\sin \left(\frac{\pi}{w+2}\right)}{2 \cos \left(\frac{\pi}{w+2}\right)+1}=\frac{2 \pi^{2}}{3 w^{3}}-\frac{4 \pi^{2}}{w^{4}}+O\left(\frac{1}{w^{5}}\right) .
$$


Note that the force is positive for all values of $w$, implying that the polymer exerts a repulsive force on the confining surfaces.

At other points, we have asymptotic expressions which will give us the asymptotic form of the force. When we are considering other values of $(a, b)$ inside $S$, we can use the form of $z_{c}$ as in (2.45), which gives:

$$
\mathcal{F}(w)=\frac{2 \pi^{2}}{3 w^{3}}-\frac{2 \pi^{2}(3 a-4 a b+3 b)}{(2 a-3)(2 b-3) w^{4}}+O\left(\frac{1}{w^{5}}\right) .
$$

This expression is positive for large $w$ and hence the force is repulsive.

Along the boundary of $S$ we first look at the point $a=3 / 2, b=1$, where $z_{c}$ is given by(2.47). Using this expression, we get:

$$
\mathcal{F}(w)=\frac{\pi^{2}}{6 w^{3}}-\frac{7 \pi^{2}}{12 w^{4}}+O\left(\frac{1}{w^{5}}\right)
$$

which is positive for large values of $w$. Also, we note that the coefficient of the leading term has changed relative to the cases inside $S$, confirming a change in behaviour of the problem from $S$ to $L$.

For other points on $L$, we can consider the expression for $z_{c}(3 / 2, b, w)$ in $(2.49)$. The resulting force is then given by:

$$
\mathcal{F}(w)=\frac{\pi^{2}}{6 w^{3}}+\frac{(4 b+3) \pi^{2}}{12(2 b-3) w^{4}}+O\left(\frac{1}{w^{5}}\right),
$$

For points outside the square $S$, we have two regimes, and hence two more expressions for $z_{c}$. For cases where $a>3 / 2$ and $a>b$, we use the form of $z_{c}$ as in (2.56) to get:

$$
\mathcal{F}(w)=-\frac{1}{2} \frac{f_{z}(a, b) q_{o}^{2 w} \log q_{o}}{q_{o}+f_{z}(a, b) q_{o}^{2 w}}+O\left(q_{o}^{3 w}\right) .
$$

By its definition, $q_{o}$ is always positive. However for all values of $a>3 / 2, q_{o}<1$. This implies that in this regime $\log q_{o}$ is negative. Also the denominator of the expression above is precisely a multiple of our definition of $q_{c}$ as in (2.54) which is always positive. As such, the nature of the force on the surfaces is determined by the sign of $f_{z}(a, b)$. Since $f_{z}(a, b)$ is zero along (2.36) it implies that the force vanishes for all $(a, b)$ along this zero force curve. Indeed, by Lemma 2.5 we know that the free energy is independent of the width along this curve and so the force is identically zero. For points below the zero force curve, we find that $f_{z}(a, b)>0$, resulting in a repulsive force between the surfaces. For the region above the curve in $(2.36)$, we find that $f_{z}(a, b)<0$, giving an attractive force. 
If instead we consider the force along the line $a=b$ with $a>3 / 2$, we use (2.61) for $z_{c}$ to get:

$$
\mathcal{F}(w)=-\frac{g_{z}(a) q_{o}^{w} \log q_{o}}{q_{o}+g_{z}(a) q_{o}^{w}}+O\left(q_{o}^{2 w}\right) .
$$

The structure of the above equation is the same as the previous case, so that the nature of the force will again be determined by the sign of $g_{z}(a)$. In this case, it turns out that $g_{z}(a)<0$ for all values of $a>3 / 2$, so that the force between the surfaces is always attractive along $a=b$ for $a>3 / 2$.

The fact that for the region outside the square $S$ the force contains a term exponential in $q_{o}$ with $q_{o}<1$, implies that the force decays exponentially in this region. As such, this region in the plane is characterized by short-ranged forces, whereas the forces inside $S$ and along its boundary are described by algebraic functions, making them long-ranged.

\subsection{Edge Model}

The edge and vertex models of Motzkin paths are closely related, as they both share the same set of steps to make up the paths. However in the edge model, the weightings $a$ and $b$ for contacts with the lower and upper surfaces are associated with the edges rather than the vertices of the path. As such, in this case, we can again define a partition function for the system via:

$$
M_{w}^{e}(a, b, z)=\sum_{\omega \in \Omega} a^{u_{1}(\omega)} b^{u_{2}(\omega)} z^{n(\omega)},
$$

where now the $u_{i}(\omega)$ 's are the numbers of edges in the appropriate surfaces.

Using the wasp-waist factorization method, we can write down an expression for $w \rightarrow \infty$, which corresponds to the case of adsorption at a single surface if the limit $w \rightarrow \infty$ is taken before the limit $n \rightarrow \infty$. For this case, the expression is:

Theorem 4. The half-plane Motzkin path generating function (2.1) factorizes as

$$
M^{e}(a, z)=\frac{1}{1-a z}\left(1+z^{2} M^{e}(1, z) M^{e}(a, z)\right),
$$

and hence

$$
M^{e}(a, z)=\frac{2}{1-2 a z+z+\sqrt{1-2 z-3 z^{2}}} .
$$


The proof is very similar to that of Theorem 1 except for the single difference of a weight $a$ which was associated with the vertex from the first down step which is not now required because of edge weights.

This expression has two singularities, $z_{1}=1 / 3$ and $z_{2}=z_{2}(a)$, where the value of $a$ for which $z_{1}=z_{2}$ is the critical value of the system. In the case of edge Motzkin paths, $a_{c}=2$, and like the vertex version of the problem, this interaction value will play an important role in determining the behaviour of the system.

Theorem 5. The following statements are equivalent.

(i) $M_{w}^{e}(a, b, z)$ is the generating function (2.70) for surface edge weighted Motzkin paths.

(ii) $M_{w}^{e}(a, b, z)$ factorizes into the

$$
M_{w}^{e}(a, b, z)=\frac{1}{1-a z}\left(1+z^{2} M_{w-1}^{e}(1, b, z) M_{w}^{v}(a, b, z)\right) .
$$

with initial (width one) generating function,

$$
M_{1}^{e}(a, b, z)=\frac{1-b z}{1-a z-b z-z^{2}+a b z^{2}} .
$$

(iii) $M_{w}^{e}(a, b, z)$ is a rational function

$$
M_{w}^{e}(a, b, z)=\frac{P_{w}^{e}(a, b, z)}{Q_{w}^{e}(a, b, z)}
$$

where the polynomials $P_{w}^{e}(a, b, z)$ and $Q_{w}^{e}(a, b, z)$ satisfy the same three term recurrence relation,

$$
T_{w+2}=(1-z) T_{w+1}-z^{2} T_{w}, \quad w \geq 0
$$

(ie replace $T_{k}$ with $P_{w}^{e}$ or $Q_{w}^{e}$ ) but with different initial conditions

$$
\begin{aligned}
& P_{0}^{e}=1, \quad P_{1}^{e}=1-b z, \quad \text { and } \\
& Q_{0}^{e}=1+z-a z-b z \quad Q_{1}^{e}=1-a z-b z-z^{2}+a b z^{2}
\end{aligned}
$$

(iv) $M_{w}^{e}(a, b, z)$ satisfies the equation

$$
M_{w}^{e}(a, b, z)=\frac{1}{1-a z-z^{2} F_{w-1}^{e}\left(M_{1}^{e}(1, b, z)\right)}, \quad w \geq 2
$$

where $F_{w}^{e}(a)$ satisfies the functional equation

$$
F_{w+1}^{e}(a)=F_{w}^{e}\left(\frac{1}{1-z-a z^{2}}\right), \quad w \geq 2
$$

with initial conditions

$$
F_{1}^{e}(a)=\frac{1}{1-z-a z^{2}} \quad \text { and } \quad F_{0}^{e}(a)=\frac{1}{1-b z} .
$$


The proof is very similar to that of Theorem 2 and we omit the details.

The procedure for determining the functional dependence of the generating functions on $w$ follows as in the vertex model. The zeros of the denominators are given by the following theorem.

Theorem 6. The zeros of the denominators of $M_{w}^{e}(a, b, z)$, that is the zeros of $Q_{w}^{e}(a, b, z)$, in the variable $q$ variable, where $z=\frac{q}{1+q+q^{2}}$ are solutions of the degree $2 w+4$ equation

$$
q^{2 w}=\frac{(a q-q-1)(b q-q-1)}{q^{2}(q-a+1)(q-b+1)} .
$$

2.2.1. Regions in the $(a, b)$ plane As for the vertex model, we study the behaviour of the edge model in several regions of the $(a, b)$ plane. The value of $a_{c}=2$ for the adsorption problem will be key in determining these regions. The regions will include the square $S$ with vertices $(0,0),(0,2),(2,0),(2,2)$, as well as the nonzero boundaries of the square and the region outside $S$. We will also exploit the symmetry with respect to interchange of $a$ and $b$ in the denominators so that we will only consider cases where $a \geq b$.

For points inside the square $S$, we first consider the point $(1,1)$. At this point, the behaviour of the edge model is identical to that of the vertex model and the result for $z(1,1, w)$ is the same as $(2.39)$. From this, we can extract the free energy via (2.4), and the asymptotics of $z_{c}(1,1, w)$ and $\kappa(1,1, w)$ in $1 / w$ will be given by (2.40) and (2.41), respectively.

At other points inside $S$ but away from $(1,1)$, we propose the same Ansatz as in (2.42), and find that in the edge version:

$$
\delta(a, b)=\frac{2(4-a-b)}{(a-2)(b-2)} .
$$

As for the vertex version, we again find that the first three coefficients of the correction terms in (2.42) are zero, and the fourth coefficient is a function of $(a, b)$, although not the same function as in the vertex case. Transforming this result by (2.25), we find that:

$$
\begin{aligned}
z_{c}(a, b, w) & =\left[\frac{1}{1+2 \cos \left(\frac{2 \pi}{2 w+\delta(a, b)}\right)}\right]\left[1+\frac{C_{4}(a, b)}{w^{4}}+O\left(\frac{1}{w^{5}}\right)\right] \\
& =\frac{1}{3}+\frac{\pi^{2}}{9 w^{2}}-\frac{2 \pi^{2}(4-a-b)}{9(a-2)(b-2) w^{3}}+O\left(\frac{1}{w^{4}}\right)
\end{aligned}
$$




$$
\begin{aligned}
\kappa(a, b, w) & =-\log \left[z_{c}(a, b, w)\right] \\
& =\log (3)-\frac{\pi^{2}}{3 w^{2}}+\frac{2 \pi^{2}(4-a-b)}{3(a-2)(b-2) w^{3}}+O\left(\frac{1}{w^{4}}\right) .
\end{aligned}
$$

The form of these results again suggests that the behaviour of the system changes when either $a=2$ or $b=2$.

We will only consider the case where $a=2$ for reasons of symmetry, and again in this situation we have two subcases. The first is at the point $(2,1)$, where the condition in (2.81) reduces to:

$$
q^{2 w}=\frac{-1}{q^{3}}
$$

The solution to this is:

$$
q_{c}(2,1, w)=\exp \left(i \frac{\pi}{2 w+3}\right)
$$

which transforms to:

$$
\begin{aligned}
z_{c}(2,1, w) & =\frac{1}{1+2 \cos \left(\frac{\pi}{2 w+3}\right)} \\
& =\frac{1}{3}+\frac{\pi^{2}}{36 w^{2}}-\frac{\pi^{2}}{12 w^{3}}+O\left(\frac{1}{w^{4}}\right) .
\end{aligned}
$$

For points along the line $L=\{(a, b) \mid a=2,0<b<2\}$ away from $(2,1)$, we utilize the same Ansatz as in (2.42) for the vertex case. In this case we find that:

$$
\delta(a, b)=\frac{(b-4)}{(b-2)}
$$

with the first non-zero correction term coming from the $w^{-4}$ term. The asymptotic forms of $z_{c}$ and $\kappa$ can be calculated as:

$$
\begin{aligned}
z_{c}(2, b, w)= & \frac{1}{3}+\frac{\pi^{2}}{36 w^{2}}-\frac{(b-4) \pi^{2}}{36(b-2) w^{3}} \\
& +\frac{\pi^{2}\left[\pi^{2}(b-2)^{2}+12(b-4)^{2}\right]}{576(b-2)^{2} w^{4}}+O\left(\frac{1}{w^{5}}\right) \\
\kappa(2, b, w)= & \log (3)-\frac{\pi^{2}}{12 w^{2}}+\frac{(b-4) \pi^{2}}{12(b-2) w^{3}} \\
& -\frac{\pi^{2}\left[\pi^{2}(b-2)^{2}+36(b-4)^{2}\right]}{576(b-2)^{2} w^{4}}+O\left(\frac{1}{w^{5}}\right)
\end{aligned}
$$

As in the vertex case, this form breaks down for the corner point of $S$. In this case, this implies that this form no longer holds at $(2,2)$, which represents a special point for the system. 
At the point $(2,2)$, we find that the condition in (2.81) simplifies to give:

$$
q^{2 w}=\frac{1}{q^{2}}
$$

From here, we can find the value of $q_{c}$ and hence $z_{c}$ in the same way that we treated $(1,1)$. However note that in this case, we no longer have the restriction that $n \neq 0$. As such, the closest singularity to the origin in this case occurs when $n=0$, which gives:

$$
q_{c}(2,2, w)=1 \quad \text { and, } \quad z_{c}(2,2, w)=\frac{1}{3} .
$$

We note that the value of $z_{c}$ is independent of $w$, and we expect that this is a point on the zero force curve for the system. One may show (in the same way as Lemma 2.5) that along the curve $a b-a-b=0$ that the dominant singularity is independent of $w$ :

$$
q_{c}=\frac{1}{a-1} \quad \text { and } \quad z_{c}=\frac{a-1}{a^{2}-a+1}
$$

for $a>b$. When $a<b$ the expressions are the same but with $b$ in place of $a$.

The analysis of the region outside $S$ follows the procedure considered for the vertex model, with similar results. In the $w \rightarrow \infty$ limit, the region where $a \geq b$, the critical value of $q$ is:

$$
q_{o}=\frac{1}{a-1}
$$

which transforms to:

$$
z_{o}=\frac{a-1}{a^{2}-a+1} \text {. }
$$

This expression for $z_{o}$ is precisely the value of $z_{c}$ for the adsorption of edge Motzkin paths at a single, impenetrable surface.

For the case of finite values of $w$, we again propose the structures of $q_{c}$ as in (2.54) and (2.59) for the case where $a>b$ and $a=b$, respectively. Note that in these expressions, the value of $q_{o}$ has changed to (2.94), as have the expressions for $f_{q}(a, b)$ and $g_{q}(a, b)$. For the edge versions, these functions are now:

$$
\begin{aligned}
& f_{q}(a, b)=\frac{a(a-2)(a+b-a b)}{(a-b)(a-1)^{4}} \text { and, } \\
& g_{q}(a, b)=-\frac{a(a-2)}{(a-1)^{4}} .
\end{aligned}
$$

In each case, the relationship between $q_{c}$ variables and the transformed $z_{c}$ variables remains the same as in (2.56) and (2.61), respectively. Note that $f_{q}(a, b)$ and $g_{q}(a, b)$ transform to $f_{z}(a, b)$ and $g_{z}(a, b)$ according to (2.57) and (2.62), respectively. 
2.2.2. Forces As with the vertex version of Motzkin paths, the force on the surfaces in the edge version of Motzkin paths follows directly from (2.63) and the appropriate expression for $z_{c}$. The results are that:

- when $a=b=1$, the result is identical to that of the vertex version of Motzkin paths, and the force is given in (2.64);

- when we consider points inside $S$, but away from $(1,1)$, we find that

$$
\mathcal{F}(w)=\frac{2 \pi^{2}}{3 w^{3}}+\frac{2 \pi^{2}(a+b-4)}{(a-2)(b-2) w^{4}}+O\left(\frac{1}{w^{5}}\right)
$$

- when $a=2$ and $b=1$, we can use the analytical form of $z_{c}$ as in (2.87) to get

$$
\mathcal{F}(w)=\frac{\pi^{2}}{6 w^{3}}-\frac{3 \pi^{2}}{4 w^{4}}+O\left(\frac{1}{w^{5}}\right) ;
$$

- for points along $L$ away from $(2,1)$ we use our series for $z_{c}$ from $(2.89)$ and find

$$
\mathcal{F}(w)=\frac{\pi^{2}}{6 w^{3}}-\frac{\pi^{2}(b-4)}{4(b-2) w^{4}}+O\left(\frac{1}{w^{5}}\right) ;
$$

For the region outside the square $S$, the arguments for obtaining the force on the surfaces are identical for both the edge and vertex versions of Motzkin paths. Although the quantitative nature of the force changes since both $f_{z}(a, b)$ and $g_{z}(a, b)$ are different in the two models, the qualitative features of the force are the same. In the case of edge Motzkin paths, the zero force curve is the collection of points satisfying:

$$
(a-1)(b-1)=1 \quad \text { or equivalently } a b-a-b=0,
$$

which includes the point $(2,2)$.

Regions outside $S$ and below this curve are characterized by a short-range repulsive force, whereas regions above this curve are associated with a short-range attractive force. The nature of this attractive force is different along the line $a=b$ than elsewhere in the plane.

\subsection{Comparisons between Dyck and Motzkin path models}

The factorization scheme which we used to derive the generating functions for both the vertex and edge versions of Motzkin paths in slits can be adapted for the vertex version of Dyck paths. This gives an alternative to the method used in (Brak et al. 2005) to derive the generating functions for Dyck paths. 
The behaviour of the edge model for Motzkin paths is very closely related to the behaviour of Dyck paths. In both cases, we can find analytical expressions for $z_{c}, \kappa$ and the force at $(1,1),\left(a_{c}, 1\right)$ and $\left(a_{c}, a_{c}\right)$, where $a_{c}$ is the critical value of the interaction for the half-plane adsorption problem. For both of these models, $a_{c}=2$. The zero force curve in each case is given by the points $(a, b)$ satisfying $a b-a-b=0$.

The generating functions for Motzkin paths in a slit can be derived directly from the corresponding Dyck path generating function. The idea is to replace every vertex in a Dyck path by either a vertex or sequence of horizontal edges, giving a set of Motzkin paths. We give the argument for the vertex model of Motzkin paths. The argument for the edge version is similar, and we not give the details. It will be convenient to associate a weight $c$ with the zeroth vertex of a Dyck path. We write $D_{w}(a, b, c, z)$ for the generating function of Dyck paths in a slit of width $w$ where $a$ is conjugate to the number of vertices (other than the zeroth vertex) in $y=0, b$ is conjugate to the number of vertices in $y=w$ and $z$ is conjugate to $n$, the number of edges. Suppose that $F_{w}(a, b, c, f)$ is the corresponding generating function where $f$ is conjugate to the number of vertices with $0<y<w$, and the other symbols retain their previous meaning. $D_{w}$ and $F_{w}$ are related by

$$
F_{w}(a, b, c, f)=D_{w}(a / f, b / f, c, f) .
$$

Next we construct Motzkin paths by replacing each vertex of a Dyck path by a vertex or a sequence of horizontal edges. We set the weight of the zeroth vertex to be unity. If $G_{w}^{v}(a, b, f)$ is the corresponding generating function for the vertex model of Motzkin paths then $G_{w}^{v}$ and $F_{w}$ are related by

$$
G_{w}^{v}(a, b, f)=F_{w}\left(\frac{a}{1-a}, \frac{b}{1-b}, \frac{1}{1-a}, \frac{f}{1-f}\right) .
$$

Finally we switch back to counting edges instead of vertices with $0<y<w$ to obtain

$$
M_{w}^{v}(a, b, z)=G_{w}^{v}(a z, b z, z) .
$$

Although this gives an alternate scheme for deriving the generating functions for the Motzkin path models the analysis given in the previous sections is still necessary. This approach does not provide a convenient mapping whereby the analysis for Dyck paths immediately gives results for Motzkin paths. 


\subsection{Paths and Pavings and Three Term Recurrences}

The appearance of three-term recurrence relations such as (2.8) and (2.75) in the context of paths-in-a-slit generating functions is a well known occurrence. Combinatorially they traditionally arise from two related sources. However, the recurrences obtained in this paper and in (Brak et al. 2005) represent a third origin. In this section we briefly explain how they differ from the former two.

The first traditional origin is the heap formulation of the Cartier-Fota commutation monoid (Cartier \& Foata 1969) due to Viennot (Viennot 1986). Here the Motzkin paths are in bijection with heaps of monomers and dimers (Viennot 1986, Bousquet-Mélou \& Rechnitzer 2002). An example of the bijection is illustrated in Figure 4. The path defines a sequence of monomers and dimers. The sequence is defined by reading the path from left to right and each time the right end of a "matching" pair of steps (shown as a line with a "box" on the end) is passed a monomer $[h]$ or dimer $[h, h+1]$ is added to the sequence, where $h$ is the $y$ coordinate of the left vertex of the path step. The sequence is then used to create a heap: the monomer $[x]$ (resp. dimer $[x, x+1]$ ) is lowered "from above" onto the heap at horizontal position $x$.
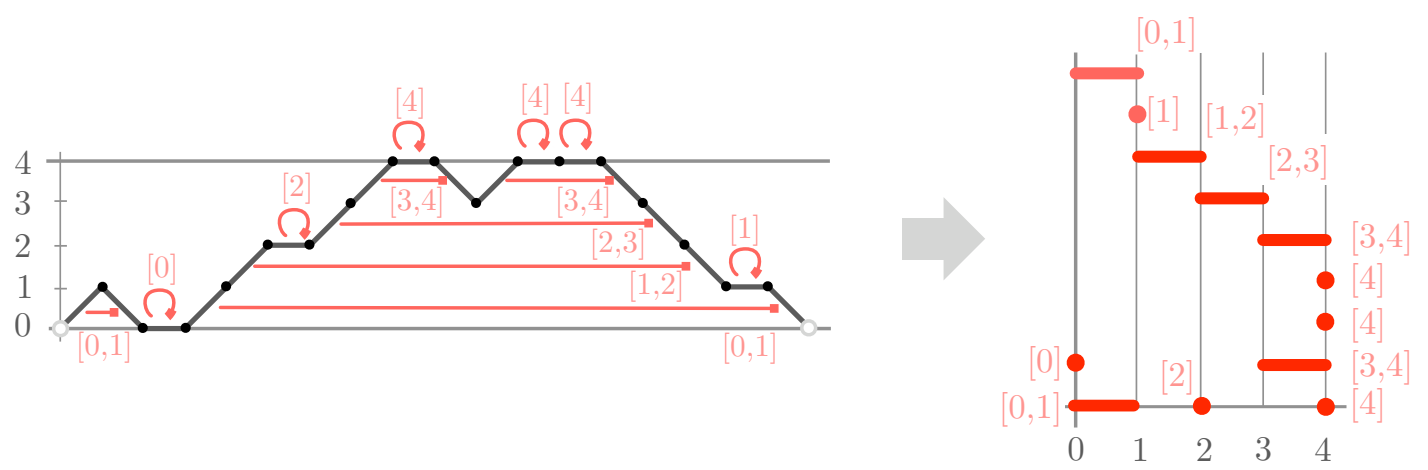

Monomer/dimer sequence $=[0,1][0][2][4][3,4][4][4][3,4][2,3][1,2][1][0,1]$

Figure 4. An example of the bijection from a Motzkin path in a strip of width four to a heap of width four.

The generating function for such heaps can, by using the heap inversion lemma, be written as a rational function. The numerator and denominator polynomials of the rational generating function generate classes of "trivial" heaps of monomers and 
dimers. Combinatorially such trivial heaps correspond to pavings of the line segment $[0, w]$ by monomers and dimers - see Figure 5 for an example. The generating function $H_{w}(z)$ for pavings by monomers and dimers on the line segment $[0, w]$ is readily shown to satisfy a three term recurrence,

$$
H_{k+1}(y)=\left(y-c_{k}\right) H_{k}(y)-\lambda_{k} H_{k-1}(y),
$$

with $H_{0}(y)=1$ and $H_{1}(y)=y-c_{0}$. A monomer on vertex $k$ has weight $-c_{k}$, a dimer on edge $k$ has weight $-\lambda_{k}$ and an isolated vertex (ie not covered by a monomer or dimer) has weight $y$. An example is shown in Figure 5. For the edge weight Motzkin

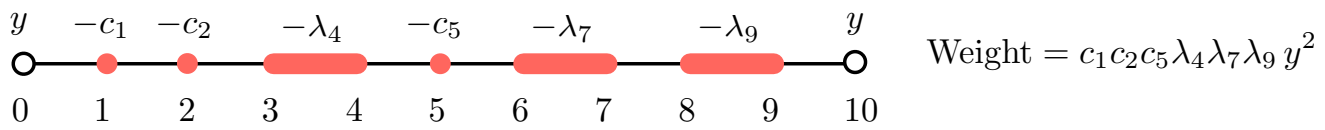

Figure 5. An example of a weighted paving of the line segment $[0,10]$ by monomers and dimers.

path problem considered in this paper one needs to have $\lambda_{k}=1$ for all $k, c_{0}=a$, $c_{w}=b$ and $c_{k}=1$ otherwise. The path generating function is then given by

$$
M_{w}^{e}(a, b, z)=\frac{H_{w-1}(1, b, 1 / z)}{z H_{w}(a, b, 1 / z)} .
$$

The reason for the replacement of $y$ by $1 / z$ and the extra factor of $z$ in the denominator is that for the path generating function we want the monomers and dimers to carry a weight of $z$ with the isolated vertices having weight one. This is achieved by replacing $H_{k}(y)$ by $z^{k} H_{k}(1 / z)$.

The second route to a three term recurrence is to write the Motzkin path generating function as

$$
M_{w}^{e}(z)=\frac{\text { cofactor }_{1,1}\left(1-z T_{w}\right)}{\operatorname{det}\left(1-z T_{w}\right)}
$$

where $T_{w}$ is the width $w$ transfer matrix for Motzkin paths - an example of a general weighted path is shown in Figure 6. Equation (2.107) may be formally derived by noting that $\left(\left(T_{w}\right)^{n}\right)_{h, h^{\prime}}$ generates paths of length $n$ (starting at height $h-1$ and ending at height $\left.h^{\prime}-1\right)$ and thus $M_{w}^{e}(z)=\sum_{n}\left(T_{w}\right)^{n} z^{n}=\left(1-z T_{w}\right)^{-1}$. The inverse matrix $\left(1-z T_{w}\right)^{-1}$ is then given by equation (2.107).

The determinant (and co-factor) which depend on the width of the slit satisfy a three term recurrence in $w$. This is the same recurrence, up to a minor 
variable change, as that obtained from the pavings, namely (2.105). In particular $\operatorname{det}\left(1-z T_{w}\right)=z^{w} H_{w}(1 / z)$. Note however, that the recurrence relations obtained from pavings, or equivalently from the determinant, are not the same as those obtained in this paper. This is seen if (2.105) is compared with say (2.76) where the $a$ and $b$ weights occur explicitly as the coefficients in (2.105), whilst for (2.76) there are no $a$ or $b$ weights in any coefficients as all the weights occur in the initial condition (2.77).

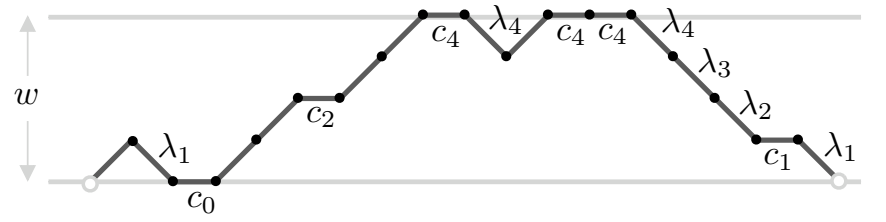

$$
T_{4}=\left(\begin{array}{cccc}
c_{0} & 1 & 0 & 0 \\
\lambda_{1} & c_{1} & 1 & 0 \\
0 & \lambda_{2} & c_{2} & 1 \\
0 & 0 & \lambda_{3} & c_{3}
\end{array}\right)
$$

Figure 6. An example of general weight Motzkin path in a slit of width four and the corresponding transfer matrix.

To simplify the remaining discussion we shall only consider the denominator polynomials $Q_{w}(z)$ given by recurrence (2.76) and the determinant polynomials $D_{w}(z)=\operatorname{det}\left(1-z T_{w}\right)=z^{w} H_{w}(1 / z)$, where $H_{w}$ satisfies $(2.105)$. The denominator of the path generating function, $M_{w}^{e}(z)$, is given by $D_{w}(z)$ if one uses the determinant or paving methods or $Q_{w}^{e}(z)$ if one uses (2.76). Note however that the prior polynomials $D_{k}$, for $k<w$, are not the same as $Q_{k}^{e}$, for $k<w$ and that they only coincide for $k=w$. Algebraically it is clear that as the recurrence (2.105) is iterated, generating the sequence $H_{1}, H_{2}, H_{3}, \ldots$ the $b$ weight is not "picked up" until $k=w$. In contrast the $b$ weight is in all the $Q_{k}^{e}$, for all $k<w$ polynomials.

Combinatorially this can be understood as follows. The sequence of generating functions $P_{k}^{e} / Q_{k}^{e}$ for $k<w$, unlike the corresponding ratio arising from the determinant/paving polynomials, (2.106), all correspond to Motzkin path generating functions (with upper and lower weight $a$ and $b$ ), but in slits of width $k<w$. Thus the three-term recurrence for $Q_{k}^{e}$ is connecting generating functions, all of which correspond to Motzkin path generating functions (with upper and lower weight $a$ and $b$ ). The ratios arising from the paving polynomial sequence do not give the $a$ and $b$ weight path generating until $k=w$. 


\section{Discussion}

We have found several equations satisfied by the generating function for Motzkin paths in a slit, with either edge weights or vertex weights associated with the lines defining the upper and lower boundaries of the slit. From these we have determined the phase diagram ( $i e$ the locus of singularities in the free energy) and force diagram for each model. The free energies and forces have been calculated exactly at several points in the $(a, b)$-plane and asymptotically (for large strip widths) elsewhere. The edge and vertex models have different critical values for the interaction parameters but the qualitative form of the phase diagram is the same. There is a zero force line in the phase diagram, determined by $(a-1)(b-1)(a+b+1)=1$ for the vertex model and $(a-1)(b-1)=1$ for the edge model, which is the same as for the Dyck path model (Brak et al. 2005).

The approach that we used to compute the path generating functions is a generalisation of the canonical factorization method. This yields three term recurrence relations, and hence sequences of orthogonal polynomials, which differ from those which are obtained by a bijection to heaps of dimers and monomers or by transfer matrix methods. Ratios of these polynomials give a sequence of the path generating functions for all slit widths unlike the heap or transfer matrix methods.

\section{Acknowledgements}

$\mathrm{RB}$ and AR would like to thank the Australian Research Council's Centre of Excellence for the Mathematics and Statistics of Complex Systems for financial assistance. SGW and AR acknowledge financial support from NSERC. 


\section{References}

Bousquet-Mélou M \& Rechnitzer A 2002 Discrete Math. 258, 235-274.

Brak R, Owczarek A L, Rechnitzer A \& Whittington S G 2005 J. Phys. A: Math. Gen. 38, 43094325.

Cartier P \& Foata D 1969 Lecture notes in Mathematics 85, 1-62.

DiMarzio E A \& Rubin R J 1971 J. Chem. Phys. 55, 4318-4336.

Hammersley J M, Torrie G M \& Whittington S G 1982 J. Phys. A: Math. Gen. 15, 539-571.

Hammersley J M \& Whittington S G 1985 J. Phys A: Math. Gen. 18, 101-111.

Janse van Rensburg E J 2000 The Statistical Mechanics of Interacting Walks, Polygons, Animals and Vesicles Oxford University Press.

Janse van Rensburg E J, Orlandini E, Owczarek A L \& Whittington A R S G 2005 J. Phys. A: Math. Gen. 38, L823-L828.

Janse van Rensburg E J, Orlandini E \& Whittington S G 2006 J. Phys. A: Math. Gen. 39, 1386913902.

Motzkin T 1948 Bull. Amer. Math. Soc. 54, 352-360.

Schröder E 1870 Z. f. Math. Phys. 15, 361-376.

Viennot X G 1986 Lecture notes in Math 1234, 321-350.

Wall F T, Seitz W A, Chin J C \& de Gennes P G 1978 Proc. Nat. Acad. Sci. 75, 2069-2070.

Wall F T, Seitz W A, Chin J C \& Mandel F 1977 J. Chem. Phys. 67, 434-438.

Wall H S 1948 Analytic Theory of Continued Fractions D. Van Nostrand New York. 